\title{
Dual-signal Amplification Strategy Aptasensor Based on Exonuclease III and Ordered Mesoporous Carbon-Gold Nanocomposites for Tetracycline Detection in Milk
}

\author{
Zengning Liu ${ }^{\# 1,2}$, Qingcui Xu ${ }^{\# 1,2}$, Jiayun $\mathrm{Fu}^{1,2}$, Zhaoqiang Shi ${ }^{1,2}$, Qingqing Yang ${ }^{1,2}$, Yemin Guo ${ }^{1,2, *}$, \\ Yanyan Zhang ${ }^{1,2}$, Xia Sun ${ }^{1,2, *}$, Zhiqiang Wang ${ }^{2,3}$ \\ ${ }^{1}$ School of Agriculture Engineering and Food Science, Shandong University of Technology, No.12 \\ Zhangzhou Road, Zibo 255049, Shandong Province, P.R. China \\ ${ }^{2}$ Shandong Provincial Engineering Research Center of Vegetable Safety and Quality Traceability, No. \\ 12 Zhangzhou Road, Zibo 255049, Shandong Province, P.R. China \\ ${ }^{3}$ College of computer science and technology, Shandong University of Technology, No.12 Zhangzhou \\ Road, Zibo 255049, Shandong Province, P.R. China \\ *E-mail: gym@sdut.edu.cn, sunxia2151@ sina.com \\ \#These authors contributed equally to this study.
}

doi: $10.20964 / 2018.08 .120$

Received: 7 May 2018 / Accepted: 23 June 2018 / Published: 5 July 2018

In this paper, a screen-printed aptasensor based on a dual-signal amplification strategy with enzymeassisted target circulation and modification with ordered mesoporous carbon (OMC)-gold nanoparticle (AuNP) nanocomposites was used to detect tetracycline (TET) in milk. Exonuclease III (Exo III), an enzyme, is known to recognize double-stranded DNA nonspecifically. To take advantage of this intrinsic property, Exo III was selected to facilitate the target circulation and amplify the signal. Furthermore, OMC and AuNP nanocomposites formed a unique sensing film with a large surface area and good electronic conductivity, amplifying the current signal for tetracycline detection with excellent sensitivity. A TET-binding aptamer was self-assembled onto the surface of screen-printed carbon electrodes (SPCEs) modified with the nanocomposites to form a sensing layer. Additionally, Exo III sheared the hybridized double chain selectively, releasing the tetracycline target. The released target and the remaining hairpin probe hybridized again. After each cycle of digestion, the target was recycled, leading to amplification. Moreover, real milk samples were directly measured after diluting the milk 3 times. The results showed that the present dual-signal amplification strategy for tetracycline analysis exhibited a detection limit of $3.0 \mu \mathrm{g} / \mathrm{L}$ with high specificity and demonstrated its applicability for detecting tetracycline residues in milk samples.

Keywords: Aptasensor; Screen-printed carbon electrodes; Ordered mesoporous carbon; Tetracycline residues; Real-time field detection 


\section{FULL TEXT}

(C) 2018 The Authors. Published by ESG (www.electrochemsci.org). This article is an open access article distributed under the terms and conditions of the Creative Commons Attribution license (http://creativecommons.org/licenses/by/4.0/). 